\title{
Avaliação da sensibilidade in vitro de isolados de Colletotrichum spp. à fungicidas
}

\author{
Amanda Letícia da SILVEIRA1*, Maria Gilmara de Oliveira SOARES ${ }^{1}$, Sarah da Silva Costa GUIMARÃES ${ }^{1}$, \\ Fernanda Dias PEREIRA ${ }^{1}$, Bruna Canabarro POZZEBON ${ }^{1}$, Eduardo ALVES ${ }^{1}$ \\ ${ }^{1}$ Departamento de Fitopatologia, Universidade Federal de Lavras, Lavras, MG, Brasil. \\ (ORCID: *; 0000-0001-7580-8854; 0000-0002-6291-0718; 0000-0002-6280-1562; 0000-0002-2719-5472; 0000-0003-3185-9291) \\ *E-mail: amandaagronomia@gmail.com (ORCID: 0000-0003-3248-9691)
}

\begin{abstract}
Recebido em: 22/10/2018; Aceito em: 28/10/2019; Publicado em: 04/02/2020.
RESUMO: Doenças pós-colheita estão entre os principais fatores que reduzem a produção da bananeira. Dentre elas a antracnose se destaca. Causada pelo fungo Colletotrichum spp. essa doença interfere na qualidade dos frutos reduzindo seu tempo de prateleira. Como medida de controle a utilização de produtos químicos tem sido a mais utilizada e com isso tem se observado a ineficiência de alguns princípios ativos. Dessa forma, o objetivo desse trabalho foi avaliar a sensibilidade micelial in vitro de isolados de Colletotrichum spp., submetidos a diferentes doses de fungicidas. $O$ delineamento experimental foi inteiramente ao acaso com 5 isolados, 3 fungicidas, 4 doses $\left(0,1 ; 1,0 ; 10 ; 100 \mathrm{mg} \mathrm{L}^{-1}\right)$ e 3 repetições de cada tratamento. Discos de micélio de cada isolado foram adicionados em placas com as doses de cada fungicida. O crescimento micelial foi medido após o micélio dos fungos das placas controle atingirem suas bordas. Foi estimada a concentração efetiva dos fungicidas capaz de inibir em $50 \%$ do crescimento micelial (EC50) e classificados em quatro categorias de eficiência. As análises estatísticas foram processadas com o programa SISVAR. Todos os fungicidas foram eficientes no controle in vitro, com destaque para o microbiológico. Para os isolados testados não foi observado resistência a nenhum dos fungicidas.
\end{abstract}

Palavras-chave: pós-colheita; Bacillus subtillis; controle químico; antracnose.

\section{Evaluation of in vitrous sensitivity of isolates of Colletotrichum spp. to fungicides}

\begin{abstract}
Post-harvest diseases are among the main factors that reduce banana production. Among them the anthracnose stands out. Caused by the fungus Colletotrichum spp. this disease interferes in the quality of the fruits reducing their shelf life. As a control measure the use of chemicals has been the most used and with this has been observed the inefficiency of some active principles. Thus, the objective of this work was to evaluate the in vitro mycelial sensitivity of Colletotrichum spp. Isolates submitted to different doses of fungicides. The experimental design was completely randomized with 5 isolates, 3 fungicides, 4 doses $(0.1,1.0,10,100 \mathrm{mg}$ $\left.\mathrm{L}^{-1}\right)$ and 3 replicates. Mycelial disks from each isolate were added on plates with the doses of each fungicide. Mycelial growth was measured after the fungal mycelium of the control plates reached their edges. The effective concentration of fungicides capable of inhibiting 50\% of mycelial growth (EC 50) and classified into four efficiency categories was estimated. Statistical analyzes were processed using the SISVAR program. All the fungicides were efficient in the in vitro control, with emphasis on the microbiological. No resistance to any of the fungicides was observed for the isolates tested.
\end{abstract}

Keywords: post-harvest; Bacillus subtillis; chemical control; anthracnose.

\section{INTRODUÇÃO}

A ocorrência de doenças na pós-colheita é um dos problemas que reduzem a qualidade e que limitam a exportação das frutas brasileiras, levando a perdas superiores a 50\% (TAVARES, 2004; TAVARES; SOUZA, 2005; MEDINA; PEREIRA, 2004), além de diminuírem o tempo de prateleira dos frutos (ANTHONY et al., 2004; SILVA et al., 2008; DEL PONTE, 2009; VENTURA; HINZ, 2002).

No caso da banana, várias podridões podem ocorrer nessa fase, porém o maior destaque é dado à antracnose, causada pelo fungo Colletotrichum spp., que se manifesta, principalmente na fruta madura (VENTURA; HINZ, 2002). A doença é caracterizada pela formação de lesões deprimidas, escuras, com o eixo maior paralelo ao eixo longitudinal e delimitada por uma margem mais clara. Sob condições de alta umidade, cobrem-se de frutificação rosada, ou acérvulos do agente patogênico. Geralmente, as lesões são superficiais, mas podem, em casos severos, com o amadurecimento da fruta, atingir a polpa (KIMATI et al., 2005).

A importância da antracnose está ligada à frequência com que aparece, principalmente, nas condições de comércio interno em que, poucos cuidados são tomados do ponto de vista de manuseio, ausência de controle químico em campo e de refrigeração. Para o controle do C. musae, devem-se considerar aspectos relacionados ao manejo adequado na pré-colheita, colheita e após a colheita (VENTURA; HINZ, 2002).

O controle desta doença pode ser por meio de medidas curativa, biológica, cultural e química, como também por meio de medidas preventivas, tais como a seleção de uma cultivar resistente, uso da redução da temperatura de armazenamento (CIMANGA et al., 2002; PUPO et al., 2003; 
SILVA et al., 2008). No entanto, a medida de controle mais utilizada são os fungicidas químicos como o imazalil e tiabendazol, os únicos liberados para uso na cultura da banana em pós-colheita (VENTUROSO et al., 2010; MINISTÉRIO DA AGRICULTURA, PECUÁRIA E ABASTECIMENTO, 2018).

A utilização incorreta da dose recomendada é um dos fatores que podem acarretar o surgimento de problemas futuros, como: variação na sensibilidade ao produto, surgimento de isolados do patógeno resistentes e, consequentemente, baixa eficiência no controle químico (GHINI; KIMATI, 2000; FRAC, 2015). Fungos fitopatogênicos, antes sensíveis a um determinado fungicida, podem apresentar isolados com menor sensibilidade ao produto ou até mesmo tornarem-se resistentes, se a aplicação for realizada de forma não recomendada. Isso pode ocorrer devido à mutação ou outro mecanismo de variabilidade do patógeno (PARREIRA et al., 2009). A resistência a fungicida é uma alteração estável e herdável de um patógeno a uma determinada dose de fungicida, resultando numa redução da sensibilidade ao produto (PEREIRA, 2009; REIS et al., 2010).

Sabe-se que espécies de Colletotrichum demonstram respostas diferentes aos fungicidas (LIYANAGE et al., 1992; JAYASINGHE; FERNANDO 1998, BROWN et al., 1996). A avaliação da eficiência de fungicidas deve ser realizada após identificação correta da espécie causadora de antracnose foliar que se tem interesse. No intuito de conhecer melhor o comportamento de isolados de Colletotrichum spp. a diferentes grupos químicos, inúmeros trabalhos são realizados em todo o mundo. Tavares; Souza (2005) testando a sensibilidade de isolado de C. gloeosporioides a diferentes fungicidas, observaram que o isolado demonstrou maior sensibilidade ao fungicida imazalil e apresentou resistência ao fungicida do grupo dos benzimidazóis o tiabendazol.

Resultados satisfatórios têm sido obtidos no controle de doenças pós-colheita (VALDEBENTO-SANHUEZA, 2001). A relação entre a diversidade de microrganismos e as suas relações antagônicas, surgem como importantes ferramentas para o controle biológico aplicado. Destaca-se como um bom agente de controle biológico o Bacillus spp. por formar endósporos e apresentar uma multiplicidade de mecanismos antagônicos. Também a produção de antibióticos é característica de algumas leveduras efetivas no controle in vitro e in vivo de doenças (BETTIOL; MORANDI, 2009).

Um dos pontos fundamentais para evitar o surgimento de patógenos resistentes a um determinado produto químico é a tomada de decisão de maneira correta de como será realizado o controle químico (PARREIRA et al., 2009). Fazer rotação de princípios ativos ou evitar o uso de fungicidas com tendência à resistência dos isolados é a principal estratégia a ser utilizada. Neste sentido, o presente trabalho teve como objetivo avaliar a sensibilidade micelial in vitro de isolados de Colletotrichum spp., submetidos a diferentes doses de fungicidas.

\section{MATERIAL E MÉTODOS}

Para a realização do teste in vitro foram utilizados quatro isolados de $C$. musae (22, 37, 55 e 64) e um isolado de $C$. aracearum (isolado 51), com alta eficiência no teste de patogenicidade, e três fungicidas (Tabela 1).
Tabela 1. Fungicidas utilizados no teste de sensibilidade in vitro de isolados de $C$. musae e $C$. aracearum, obtidos de frutos de banana.

Table 1. Fungicides used the in vitro sensitivity test $\mathrm{f} C$. musae and C. aracearum, from banana fruits.

\begin{tabular}{|c|c|c|c|}
\hline $\begin{array}{c}\text { Ingrediente } \\
\text { Ativo }\end{array}$ & $\begin{array}{c}\text { Nome } \\
\text { Comercial } \\
\end{array}$ & $\begin{array}{c}\text { Grupo } \\
\text { Químico }\end{array}$ & $* \mathrm{CIA}$ \\
\hline $\begin{array}{l}\text { Bacillus subtilis } \\
\text { linhagem QST } \\
713\end{array}$ & Serenade & $\begin{array}{c}\text { Fungicida } \\
\text { microbiológico }\end{array}$ & $13,6 \mathrm{~g} \mathrm{~L}^{-}$ \\
\hline Imazalil & Magnate & Imidazol & $500 \mathrm{~g} \mathrm{~L}^{-1}$ \\
\hline Tiabendazol & Tecto & Benzimidazol & $485 \mathrm{~g} \mathrm{~L}^{-1}$ \\
\hline
\end{tabular}

*Concentração de ingrediente ativo.

Para a determinação da EC50 (concentração efetiva inibitória de $50 \%$ do crescimento micelial) os fungicidas foram diluídos em série a partir de uma solução estoque até a concentração desejada.

O delineamento experimental foi inteiramente casualizado (DIC) com cinco isolados, dois fungicidas químicos e um microbiológico, quatro doses de fungicida $\left(0,1 ; 1,0 ; 10 ; 100 \mathrm{mg} \mathrm{L}^{-1}\right)$ e a testemunha sem fungicida e três repetições de cada tratamento. Cada fungicida foi adicionado ao meio $\mathrm{BDA}$ a $45^{\circ} \mathrm{C}$ e distribuído em placas de Petri de nove $\mathrm{cm}$ de diâmetro. Em seguida, discos de micélio (seis $\mathrm{mm}$ de diâmetro) de cultura pura de sete dias dos isolados foram colocados no centro das placas, que posteriormente foram incubadas em BOD $25^{\circ} \mathrm{C} \pm 1^{\circ} \mathrm{C}$ e fotoperíodo de $12 \mathrm{~h}$.

As avaliações constaram da medição do crescimento micelial com a utilização de um paquímetro, por meio da média dos diâmetros perpendiculares entre si (diâmetro médio de colônia). Neste caso a testemunha foi desconsiderada. As avaliações foram realizadas quatro dias após a inoculação, quando as colônias das placas controle, atingiram a borda das placas. Foi estimada a concentração efetiva do fungicida capaz de inibir em $50 \%$ do crescimento micelial (EC50). O EC50 foi calculado com base na porcentagem de inibição de crescimento de cada isolado nas diferentes concentrações dos fungicidas quando comparados ao crescimento nas placas sem fungicidas (testemunha). Posteriormente foi realizada uma regressão linear simples entre a porcentagem de inibição e o log de cada dose para linearização dos valores.

Após o cálculo da EC50, os fungicidas foram classificados em quatro categorias de eficiência, de acordo com a escala de Edgington et al. (1971), citada por Tavares (2005), em que: EC50 < $1 \mathrm{mg} \mathrm{L}^{-1}$ alta eficiência (AE); EC50 1 - $10 \mathrm{mg} \mathrm{L}^{-1}$ moderada eficiência (ME); EC50 10 - $50 \mathrm{mg} \mathrm{L}$ ${ }^{1}$ baixa eficiência (BE) e EC50 > 50 $\mathrm{mg} \mathrm{L}^{-1}$ insensibilidade (I).

As análises estatísticas foram processadas com o auxílio do programa SISVAR versão 5.6. (FERREIRA, 2017). Quando houve diferença estatística foi realizado o agrupamento pelo teste Scott-Knott (entre fungicidas e isolados) ou regressão (entre as doses).

\section{RESULTADOS}

Os fungicidas tiabendazol e imazalil apresentaram EC 50 igual a 1,02 e $2,8 \mathrm{mg} \mathrm{L}^{-1}$ respectivamente, enquanto o fungicida microbiológico Bacillus subtilis (Bs) apresentou EC 50 igual a $0 \mathrm{mg} \mathrm{L}^{-1}$. Esse valor $0 \mathrm{mg} \mathrm{L}^{-1}$ encontrado para o Bs ocorreu porque o fungo não cresceu na presença de qualquer uma das doses testadas do fungicida e na testemunha o crescimento do fungo foi vigoroso. Como o cálculo da EC 50 foi realizado comparando com a testemunha, o valor foi 
zero. De acordo com a classificação pela escala de Edgington et al. (1971) o produto a base de Bs foi considerado de alta eficiência (EC50 $<1 \mathrm{mg} \mathrm{L}^{-1}$ ) e os demais fungicidas de moderada eficiência $\left(\mathrm{EC} 50 \leq 10 \mathrm{mg} \mathrm{L}^{-1}\right)$.

Os resultados da análise da variância dos dados de crescimento micelial de Colletotrichum spp, indicam que existe diferença significativa entre os tratamentos, entre cada fator estudado e a interação dupla e tripla (Tabela 2).

Todos os isolados testados tiveram o crescimento micelial reduzido na presença dos fungicidas, independente do princípio ativo utilizado. A resposta de sensibilidade variou de acordo com o fungicida testado. Foram realizados desdobramentos dos valores médios do crescimento micelial (mm) em função dos fungicidas para as combinações entre os isolados dentro de cada dose aplicada (Tabelas 3 a 6).

Na tabela 3 são mostrados os valores médios do diâmetro das colônias para a dose $0,1 \mathrm{mg} \mathrm{L}^{-1} \mathrm{e}$ isolados. Quando fixada a dose $0,1 \mathrm{mg} \mathrm{L}^{-1}$, há destaque do fungicida $\mathrm{Bs}$, que proporcionou os menores valores de crescimento micelial independente do isolado, sendo superior no controle quando comparado com os demais.

Tabela 2. Resumo da análise de variância dos dados de crescimento micelial em função dos fungicidas, doses, isolados e suas interações. Tabel 2. Abstract of the analysis of variance of mycelial growth data as a function of fungicides, doses, isolates and their interactions.

\begin{tabular}{lcc}
\hline \multicolumn{1}{c}{ FV } & GL & QM \\
\hline Fungicida & 2 & $13567.98^{* *}$ \\
Dose & 3 & $19501.26^{* *}$ \\
Isolado & 4 & $1067.40^{* *}$ \\
Fungicida x Dose & 6 & $3814.04^{* *}$ \\
Fungicida x Isolado & 8 & $118.48^{* *}$ \\
Dose x Isolado & 12 & $407.81^{* *}$ \\
Fungicida x Dose x Isolado & 24 & $122.72^{* *}$ \\
Erro & 164 & 10,33 \\
\hline Média (mm) & 22,51 & \\
CV (\%) & 14,28 & \\
\hline$* *$ Significativo a 1\% de probabilidade pelo teste F &
\end{tabular}

Tabela 3. Diâmetro médio das colônias $(\mathrm{mm})$ de $C$. musae e $C$. aracearum em função dos fungicidas na dosagem de $0,1 \mathrm{mg} \mathrm{L}^{-1}$ para cada isolado.

Tabel 3. Mean diameter of the colonies $(\mathrm{mm})$ of $C$. musae e $C$. aracearum as fungicides at the dosage of $0,1 \mathrm{mg} \mathrm{L}^{-1}$ for each isolate.

\begin{tabular}{lccccc}
\hline \multirow{2}{*}{ Fungicidas } & \multicolumn{5}{c}{ Isolados } \\
\cline { 2 - 6 } & 22 & 51 & 64 & 37 & 55 \\
\hline Bacillus subtilis & $12,57 \mathrm{a}$ & $13,66 \mathrm{a}$ & 10,93 & $9,09 \mathrm{a}$ & $7,12 \mathrm{a}$ \\
Tiabendazol & $42,63 \mathrm{~b}$ & $73,30 \mathrm{~b}$ & $32,75 \mathrm{~b}$ & $58,11 \mathrm{~b}$ & $34,75 \mathrm{~b}$ \\
Imidazol & $60,11 \mathrm{c}$ & $82,70 \mathrm{c}$ & $57,62 \mathrm{c}$ & $62,64 \mathrm{c}$ & $36,86 \mathrm{~b}$ \\
\hline
\end{tabular}

Médias seguidas pela mesma letra na vertical pertencem a um mesmo grupo pelo teste Scott-Knott a 5\%.

Na Tabela 4 estão os valores médios do diâmetro das colônias quando aplicado a dose de $1 \mathrm{mg} \mathrm{L}^{-1}$. Nota-se que os produtos tiabendazol e Bs foram superiores em todos os isolados testados, exceto para o 22 (neste caso apenas o tiabendazol se destacou).

Houve uma maior eficiência do Bs quando aplicou-se menor dose, confirmado com dados da Tabela 5, em que aplicando dose de $10 \mathrm{mg} \mathrm{L}^{-1}$ as maiores médias foram para o tiabendazol e imidazol. Na dose de $100 \mathrm{mg} \mathrm{L}^{-1}$ observa-se que todos os fungicidas foram eficientes no controle do crescimento micelial independente do isolado, exceto para o isolado 51 (Tabela 6).

Nas Figuras de 1 a 5 estão os valores médios dos diâmetros das colônias em função das doses de imidazol para os diferentes isolados. Observa-se que quando se aplica o produto ocorre uma queda acentuada até aproximadamente $20 \mathrm{mg} \mathrm{L^{-1 }}$. Após este valor ocorre um decréscimo do diâmetro menos acentuado, evidenciando que o controle foi efetivo em menores doses.

No desdobramento das doses de Bs dentro de cada isolado houve efeito significativo $(\mathrm{P}<0,05)$ apenas para $\mathrm{O}$ isolado 22. Houve tendência similar ao verificado para imidazol, ou seja, queda da variável acentuada até $20 \mathrm{mg} \mathrm{L}^{-1}$ (Figura 6). Para o fungicida tiabendazol houve queda no diâmetro das colônias em doses próximas de 5 a $10 \mathrm{mg} \mathrm{L}^{-1}$, independente do isolado (Figura 7 a 11).

Tabela 4. Diâmetro médio das colônias $(\mathrm{mm})$ de $C$. musae e $C$. aracearum $\mathrm{em}$ função dos fungicidas na dosagem 1,0 $\mathrm{mg} \mathrm{L}^{-1}$ para cada isolado.

Tabel 4. Mean diameter of the colonies $(\mathrm{mm})$ of $C$. musae e $C$. aracearum as fungicides at the dosage of $1,0 \mathrm{mg} \mathrm{L}^{-1}$ for each isolate.

\begin{tabular}{lccccc}
\hline \multirow{2}{*}{ Fungicidas } & \multicolumn{5}{c}{ Isolados } \\
\cline { 2 - 6 } & 22 & 51 & 64 & 37 & 55 \\
\hline Bacillus subtilis & $11,16 \mathrm{~b}$ & $11,69 \mathrm{a}$ & $6,78 \mathrm{a}$ & $8,78 \mathrm{a}$ & $5,00 \mathrm{a}$ \\
Tiabendazol & $5,00 \mathrm{a}$ & $10,98 \mathrm{a}$ & $7,72 \mathrm{a}$ & $5,00 \mathrm{a}$ & $5,00 \mathrm{a}$ \\
Imidazol & $45,51 \mathrm{c}$ & $63,99 \mathrm{~b}$ & 52,03 & $40,53 \mathrm{~b}$ & 44,89 \\
\hline
\end{tabular}

Médias seguidas pela mesma letra na vertical pertencem a um mesmo grupo pelo teste Scott-Knott a 5\%.

Tabela 5. Diâmetro médio das colônias $(\mathrm{mm})$ de $C$. musae e $C$. aracearum em função dos fungicidas na dosagem de $10,0 \mathrm{mg} \mathrm{L}^{-1}$ para cada isolado.

Tabel 5. Mean diameter of the colonies (mm) of C. musae e $C$. aracearum as fungicides at the dosage of $10,0 \mathrm{mg} \mathrm{L}^{-1}$ for each isolate.

\begin{tabular}{lccccc}
\hline \multirow{2}{*}{ Fungicidas } & \multicolumn{5}{c}{ Isolados } \\
\cline { 2 - 6 } & 22 & 51 & 64 & 37 & 55 \\
\hline Bacillus subtilis & $5,28 \mathrm{a}$ & $11,53 \mathrm{~b}$ & $5,00 \mathrm{a}$ & $7,85 \mathrm{a}$ & $5,00 \mathrm{a}$ \\
Tiabendazol & $5,00 \mathrm{a}$ & $7,33 \mathrm{a}$ & $5,00 \mathrm{a}$ & $5,00 \mathrm{a}$ & $5,00 \mathrm{a}$ \\
Imidazol & $5,00 \mathrm{a}$ & $5,00 \mathrm{a}$ & $5,00 \mathrm{a}$ & $11,80 \mathrm{~b}$ & $5,00 \mathrm{a}$ \\
\hline
\end{tabular}

Médias seguidas pela mesma letra na vertical pertencem a um mesmo grupo pelo teste Scott-Knott a 5\%.

Tabela 6. Diâmetro médio das colônias (em $\mathrm{mm}$ ) de $C$. musae e $C$. aracearum em função dos fungicidas na dosagem de $100 \mathrm{mg} \mathrm{L}^{-1}$ para cada isolado.

Tabel 6. Mean diameter of the colonies $(\mathrm{mm})$ of $C$. musae e $C$. aracearum as fungicides at the dosage of $100 \mathrm{mg} \mathrm{L}^{-1}$ for each isolate.

\begin{tabular}{lccccc}
\hline \multirow{2}{*}{ Fungicidas } & \multicolumn{5}{c}{ Isolados } \\
\cline { 2 - 6 } & 22 & 51 & 64 & 37 & 55 \\
\hline Bacillus subtilis & $5,01 \mathrm{a}$ & $5,00 \mathrm{a}$ & $5,00 \mathrm{a}$ & $6,60 \mathrm{a}$ & $5,00 \mathrm{a}$ \\
Tiabendazol & $5,00 \mathrm{a}$ & $8,10 \mathrm{a}$ & $5,00 \mathrm{a}$ & $5,00 \mathrm{a}$ & $5,00 \mathrm{a}$ \\
Imidazol & $5,00 \mathrm{a}$ & $12,16 \mathrm{~b}$ & $5,00 \mathrm{a}$ & $5,00 \mathrm{a}$ & $5,00 \mathrm{a}$
\end{tabular}

Médias seguidas pela mesma letra na vertical pertencem a um mesmo grupo pelo teste Scott-Knott a 5\%.

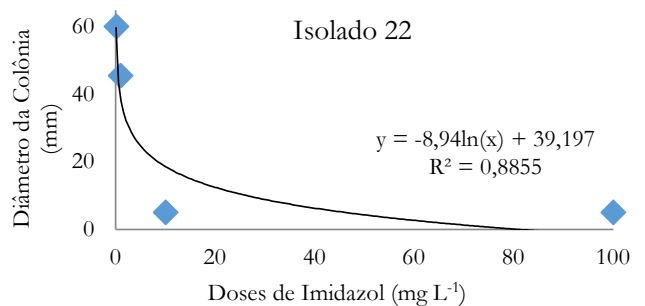

Figura 1. Diâmetro médio das colônias $(\mathrm{mm})$ de $C$. musae e $C$. aracearum em função das doses do fungicida imidazol aplicadas no isolado 22.

Figure 1. Mean diameter of the colonies $(\mathrm{mm})$ of C. musae and C. aracearum as a function of the doses of the imidazole fungicide applied in the isolate 22 . 


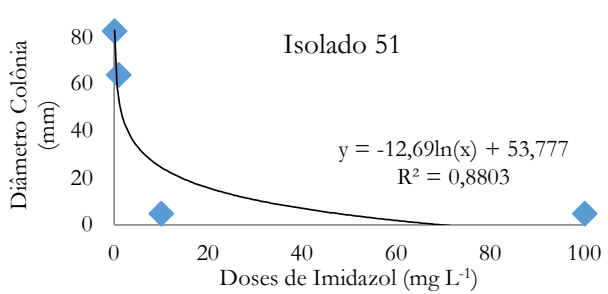

Figura 2 - Diâmetro médio das colônias ( $\mathrm{mm}$ ) de C. musae e $C$. aracearum em função das doses do fungicida imidazol aplicadas no isolado 51 .

Figure 2 - Mean diameter of the colonies ( $\mathrm{mm}$ ) of C. musae and $C$. aracearum as a function of the doses of the imidazole fungicide applied in the isolate 51 .

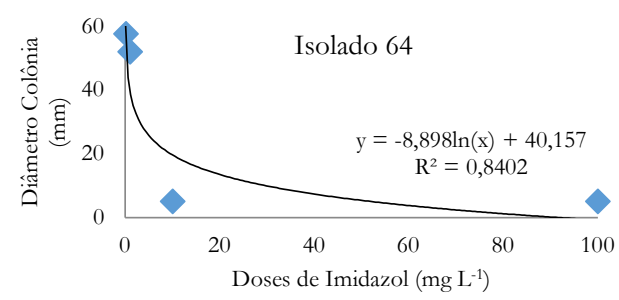

Figura 3. Diâmetro médio das colônias ( $\mathrm{mm})$ de $C$. musae e $C$. aracearum em função das doses do fungicida imidazol aplicadas no isolado 64.

Figure 3. Mean diameter of the colonies $(\mathrm{mm})$ of C. musae and $C$. aracearum as a function of the doses of the imidazole fungicide applied in the isolate 64 .

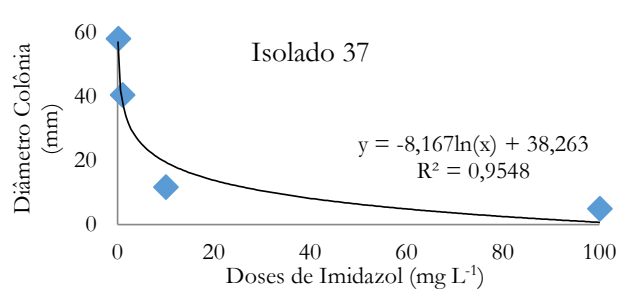

Figura 4. Diâmetro médio das colônias ( $\mathrm{mm}$ ) de C. musae e C. aracearum em função das doses do fungicida imidazol aplicadas no isolado 37. Figure 4. Mean diameter of the colonies $(\mathrm{mm})$ of $C$. musae and $C$. aracearum as a function of the doses of the imidazole fungicide applied in the isolate 37 .

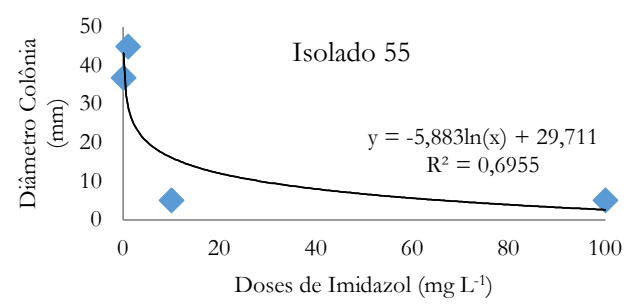

Figura 5. Diâmetro médio das colônias $(\mathrm{mm})$ de C. musae e C. aracearum em função das doses do fungicida imidazol aplicadas no isolado 55. Figure 5. Mean diameter of the colonies $(\mathrm{mm})$ of $C$. musae and $C$. aracearum as a function of the doses of the imidazole fungicide applied in the isolate 55 .

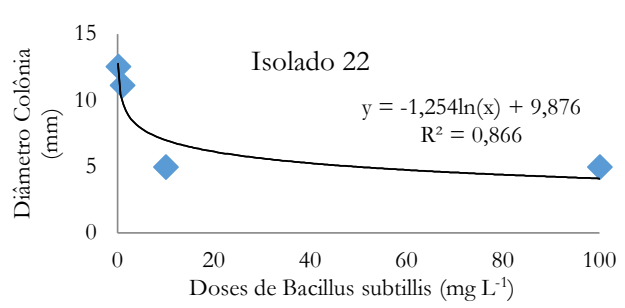

Figura 6. Diâmetro médio das colônias ( $\mathrm{mm}$ ) de C. musae em função das doses do fungicida Bacillus subtillis.

Figure 6. Mean diameter of the colonies $(\mathrm{mm})$ of C. musae and as a function of the doses of the fungicide Bacillus subtillis.

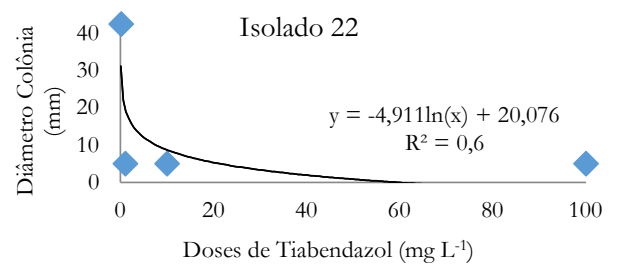

Figura 7. Diâmetro médio das colônias ( $\mathrm{mm}$ ) de $C$. musae e $C$. aracearum em função das doses do fungicida Tiabendazol aplicadas nos isolado 22. Figure 7. Mean diameter of the colonies $(\mathrm{mm})$ of C. musae and C. aracearum as a function of the doses of the Thiabendazole fungicide applied in the isolate 22 .

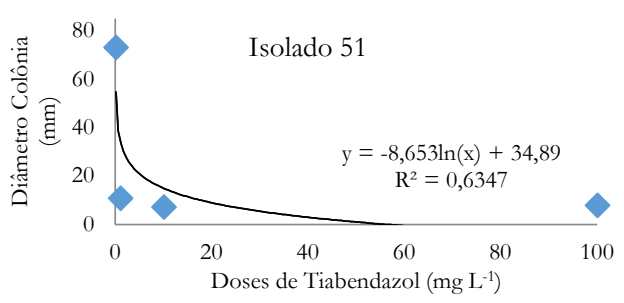

Figura 8. Diâmetro médio das colônias $(\mathrm{mm})$ de $C$. musae e C. aracearum em função das doses do fungicida Tiabendazol aplicadas no isolado 51. Figure 8. Mean diameter of the colonies ( $\mathrm{mm})$ of $C$. musae and $C$. aracearum as a function of the doses of the Thiabendazole fungicide applied in the isolate 51.

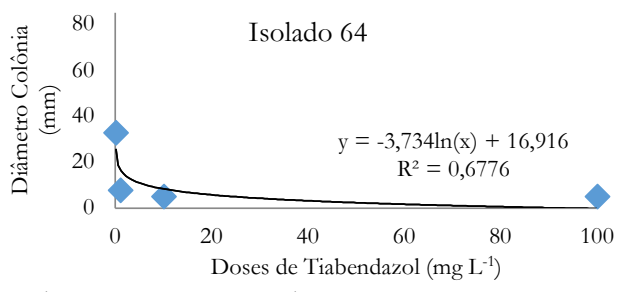

Figura 9. Diâmetro médio das colônias $(\mathrm{mm})$ de $C$. musae e $C$. aracearum em função das doses do fungicida Tiabendazol aplicadas no isolado 64. Figure 9. Mean diameter of the colonies $(\mathrm{mm})$ of C. musae and $C$ aracearum as a function of the doses of the Thiabendazole fungicide applied in the isolate 64 .

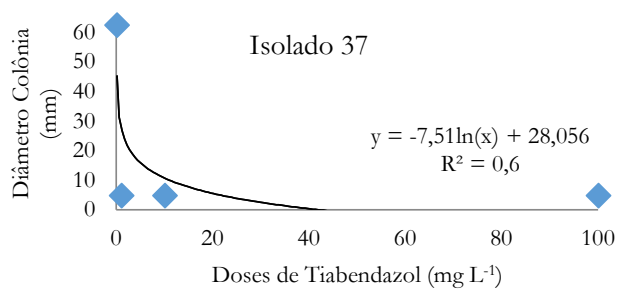

Figura 10. Diâmetro médio das colônias $(\mathrm{mm})$ C. musae e C. aracearum em função das doses do fungicida Tiabendazol aplicadas no isolado 37. Figure 10. Mean diameter of the colonies (mm) of C. musae and $C$. aracearum as a function of the doses of the Thiabendazole fungicide applied in the isolate 37 .

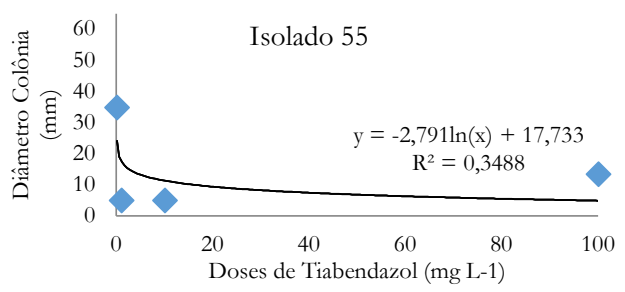

Figura 11. Diâmetro médio das colônias (mm) de C. musae e C. aracearum em função das doses do fungicida Tiabendazol aplicadas no isolado 55. Figure 11. Mean diameter of the colonies $(\mathrm{mm})$ of $C$. musae and $C$. aracearum as a function of the doses of the Thiabendazole fungicide applied in the isolate 55 . 
Nas Tabelas 7, 8 e 9 estão os valores médios dos diâmetros das colônias em função dos isolados para as diferentes doses dentro dos fungicidas imidazol, Bs e tiabendazol, respectivamente.

Quando aplicado o fungicida imidazol na dose $0,1 \mathrm{mg} \mathrm{L}$ 1 (Tabela 7), o isolado 55 foi o mais suscetível a esse fungicida seguido do 37, 64 e 22, e o isolado mais resistente foi o 51.

Para o fungicida Bacillus subtillis aplicado na dose $0,1 \mathrm{mgL}$ 1 (Tabela 8) não houve diferença estatística para os isolados testados. $\mathrm{O}$ isolado 51 se destacou negativamente, pois nas doses de 1 e $10 \mathrm{mg}$ L- ele apresentou maior diâmetro médio das colônias e só foi controlado na dose de $100 \mathrm{mg} \mathrm{L}^{-1}$.

Tabela 7. Diâmetro médio das colônias $(\mathrm{mm})$ de C. musae e $C$. aracearum em função dos isolados quando aplicado o fungicida imidazol em diferentes doses.

Table 7. Mean diameter of the colonies $(\mathrm{mm})$ of $C$. musae and $C$ aracearum as a function of the isolates when applied the imidazole fungicide at different doses.

\begin{tabular}{ccccc}
\hline \multirow{2}{*}{ Isolados } & \multicolumn{4}{c}{ Doses mg L-1 } \\
\cline { 2 - 5 } & 0,1 & 1,0 & 10 & 100 \\
\hline 22 & $60,11 \mathrm{~b}$ & $45,51 \mathrm{~b}$ & $5,00 \mathrm{a}$ & $5,00 \mathrm{a}$ \\
51 & $82,70 \mathrm{c}$ & $63,99 \mathrm{~d}$ & $5,00 \mathrm{a}$ & $12,16 \mathrm{~b}$ \\
64 & $57,82 \mathrm{~b}$ & $52,03 \mathrm{c}$ & $5,00 \mathrm{a}$ & $5,00 \mathrm{a}$ \\
37 & $58,11 \mathrm{~b}$ & $40,53 \mathrm{a}$ & $11,80 \mathrm{~b}$ & $5,00 \mathrm{a}$ \\
55 & $36,86 \mathrm{a}$ & $44,89 \mathrm{~b}$ & $5,00 \mathrm{a}$ & $5,00 \mathrm{a}$ \\
\hline
\end{tabular}

Médias seguidas pela mesma letra na vertical pertencem a um mesmo grupo pelo teste ScottKnott a 5\%.

Tabela 8. Diâmetro médio das colônias $(\mathrm{mm})$ de C. musae e $C$. aracearumem função dos isolados quando aplicado o fungicida Bacillus subtillis em diferentes doses.

Table 8. Mean diameter of the colonies (mm) of C. musae and $C$. aracearum as a function of the isolates when applied the Bacillus subtillis at different doses.

\begin{tabular}{ccccc}
\hline \multirow{2}{*}{ Isolados } & \multicolumn{4}{c}{ Doses $\left(\mathrm{mg} \mathrm{L}^{-1}\right)$} \\
\cline { 2 - 5 } & 0,1 & 1,0 & 10 & 100 \\
\hline 22 & $12,57 \mathrm{a}$ & $11,16 \mathrm{~b}$ & $5,28 \mathrm{a}$ & $5,01 \mathrm{a}$ \\
51 & $13,66 \mathrm{a}$ & $11,69 \mathrm{~b}$ & $11,53 \mathrm{~b}$ & $5,00 \mathrm{a}$ \\
64 & $10,93 \mathrm{a}$ & $6,78 \mathrm{a}$ & $5,00 \mathrm{a}$ & $5,00 \mathrm{a}$ \\
37 & $9,09 \mathrm{a}$ & $8,78 \mathrm{~b}$ & $7,85 \mathrm{a}$ & $6,60 \mathrm{a}$ \\
55 & $7,12 \mathrm{a}$ & $5,00 \mathrm{a}$ & $5,00 \mathrm{a}$ & $5,00 \mathrm{a}$ \\
\hline
\end{tabular}

Médias seguidas pela mesma letra na vertical pertencem a um mesmo grupo pelo teste ScottKnott a 5\%.

Já para o fungicida tiabendazol, quando se aplicou doses acima de $1 \mathrm{mg} \mathrm{L}^{-1}$ não ocorreu diferença do diâmetro médio das colônias em função dos isolados (Tabela 9). Os isolados 64 e 37 apresentaram menores médias para a dose $0,1 \mathrm{mg} \mathrm{L}$ 1

Tabela 9. Diâmetro médio das colônias $(\mathrm{mm})$ de $C$. musae e $C$. aracearum em função dos isolados quando aplicado o fungicida tiabendazol em diferentes doses.

Table 9. Mean diameter of the colonies ( $\mathrm{mm}$ ) of C. musae and C. aracearum as a function of the isolates when applied the Thiabendazole at different doses.

\begin{tabular}{ccccc}
\hline \multirow{2}{*}{ Isolados } & \multicolumn{4}{c}{ Doses $\left(\mathrm{mg} \mathrm{L}^{-1}\right)$} \\
\cline { 2 - 5 } & 0,1 & 1,0 & 10 & 100 \\
\hline 22 & $42,69 \mathrm{~b}$ & $5,00 \mathrm{a}$ & $5,00 \mathrm{a}$ & $5,00 \mathrm{a}$ \\
51 & $73,30 \mathrm{~d}$ & $10,93 \mathrm{a}$ & $7,33 \mathrm{a}$ & $8,10 \mathrm{a}$ \\
64 & $32,75 \mathrm{a}$ & $7,77 \mathrm{a}$ & $5,00 \mathrm{a}$ & $5,00 \mathrm{a}$ \\
37 & $62,64 \mathrm{c}$ & $5,00 \mathrm{a}$ & $5,00 \mathrm{a}$ & $5,00 \mathrm{a}$ \\
55 & $34,75 \mathrm{a}$ & $5,00 \mathrm{a}$ & $5,00 \mathrm{a}$ & $5,00 \mathrm{a}$ \\
\hline
\end{tabular}

Médias seguidas pela mesma letra na vertical pertencem a um mesmo grupo pelo teste ScottKnott a 5\%.

\section{DISCUSSÃO}

Os isolados de C. musae e C. aracearum utilizados no teste de sensibilidade foram selecionados por apresentarem alta eficiência no teste de patogenicidade.

Os resultados encontrados neste estudo diferiram dos encontrados por Tavares; Souza (2005) onde em trabalho realizado com isolados de C. gloeosporioides obtidos de frutos de mamão os autores observaram que o fungicida tiabendazol, demonstrou baixa eficiência no controle do $C$. gloeosporioides, sendo a EC 50 igual a 33,30. Porém para o fungicida Imazalil (inibidor da síntese de esteróis) o resultado observado pelos autores coincide com os encontrados nesse estudo, que mostra que os isolados testados são sensíveis a esse fungicida. Resultados semelhantes foram registrados com Colletotrichum gloeosporioides em Citrus (GOES, 1995) e Colletotrichum acutatum em morango (KOSOSKI et al., 2001).

A tolerância de espécies do fungo aos fungicidas pertencentes ao grupo dos benzimidazóis foi observada por Lapeyre de Bellayre; Dubois (1997) e Khan et al. (2001), em isolados de Colletotrichum musae oriundos de fruto de banana. Isso provavelmente deve-se ao uso contínuo deste fungicida pelos produtores para o controle do patógeno. Porém, nesse estudo os isolados testados não apresentaram resistência a esse princípio ativo, sendo que sua EC 50 foi considerada moderada, este fato pode ter ocorrido devido os frutos obtidos neste estudo não terem sido colhidos de áreas onde a pulverização com produtos químicos seja intensa, pois sabe-se que a pressão de seleção ocorre devido ao uso contínuo de mesmo princípio ativo.

Já para o fungicida a base do agente biológico Bs, Stracieri et al. (2015) estudando Colletotrichum spp. em isolados de manga consideraram este fungicida como de baixa eficiência diferindo do resultado encontrado nesse trabalho para isolados de Colletotrichum de banana, em que o fungicida biológico foi classificado como altamente eficiente in vitro.

O Bs é um fungicida microbiológico que possui múltiplos modos de ação. Os lipopeptídeos produzidos pelo Bacillus subtilis QST713 presentes na formulação atuam na membrana celular das estruturas reprodutivas do fungo, provocando sua deformação e produzindo rupturas. É usado em pulverização preventiva no controle de doenças (STRACIERI, 2015).

Mahadtanapuk et al. (2007) testando a ação de três espécies dos bacilos (Bacillus licheniformis, Bacillus amyloliquefaciens e Bacillus subtilis) sobre a germinação de Colletotrichum musae, nas concentrações de $1 \times 10^{8}, 2 \times 10^{8}, 3 \times 10^{8}$ e $4 \times 10^{8}$ (ufc $\mathrm{mL}^{-1}$ ) comprovaram que, para as concentrações

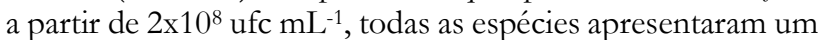
absoluto efeito inibitório $(100 \%)$ sobre a germinação de conídios em comparação ao controle (2\%). Provavelmente os demais fungicidas testados necessitam de doses maiores para serem eficientes.

Outros trabalhos mostraram resultados contrários aos verificados. Tavares e Souza (2005) para controle in vitro de Colletotrichum gloeosporioides do mamoeiro, observaram que o fungicida tiabendazol foi ineficiente no controle do patógeno e já imazalil foi altamente eficiente. Da mesma forma resultados semelhantes foram registrados com Colletotrichum gloeosporioides em Citrus (GOES, 1995) e Colletotrichum acutatum em morango (KOSOSKI et al., 2001), em que os isolados testados foram mais sensíveis ao imazalil.

No Brasil, Kupper; Gimenes-Fernandes (2002) estudaram a potencialidade antagonística de 64 isolados de $\mathrm{B}$. subtilis a C. acutatum in vitro e em flores destacadas de lima ácida, 'Tahiti'. Segundo os autores, todos os isolados 
produziram metabólitos capazes de inibir o crescimento micelial do fitopatógeno e muitos deles deram 100\% de controle da doença em flores destacadas de lima ácida "Tahiti".

\section{CONCLUSÕES}

Dentre os fungicidas testados o fungicida microbiológico foi considerado de alta eficiência e o imidazol e o tiabendazol foram considerados de eficiência moderada.

Todos os fungicidas testados foram eficientes para o controle in vitro de C. musae e C. aracearum.

Em doses menores o fungicida a base do agente biológico Bacillus subtilis foi mais eficiente que os outros fungicidas testados.

Não foi observado resistência a nenhum dos fungicidas utilizados.

\section{REFERÊNCIAS}

ANTHONY, S.; ABEYWICKRAMA, K.; DAYANANDA, R.; WIJERATNAM, S.; ARRABEWELA, L. Fungal pathogens associated with banana fruit in Siri Lanka, and their treatment with essential oils. Mycopathologia, Den Haag, v. 157, n 1, p. 99-97, 2004. DOI: https://dx.doi.org/10.1023/B:MYCO.0000012226.9562 8.99

BETTIOL, W.; MORANDI, M. A. B. Biocontrole de doenças de plantas: uso e perspectivas. Jaguariúna: Embrapa Meio Ambiente, 2009. 341 p.

BROWN, A. E.; SREENIVASAPRASAD, S.; TIMMER, L. W. Molecular characterization of slow growing orange and key lime anthracnose strains of Colletotrichum from citrus as C. acutatum. Phytopathology, Saint Paul, v. 86, n. 5, p. 523-527, 1996.

CIMANGA, K.; APERS. S.; BRUYNE, T.; MIERT, S. V.; HERMANS, N.; TOTTÉ, J.; PIETERS, L.; VLIETINCK, A. J.; KAMBU, K.; TONA, L. Chemical composition and antifungal activity of essential oils of some aromatic plants growing in the Democratic Republic of Congo. Journal of Essential Oil Research, v. 14, p.382-387, September/October, 2002.

DEL PONTE, E. M. (Ed.) Fitopatologia.net - herbário virtual. Departamento de Fitossanidade. Agronomia, UFRGS. 2009. Disponível em: <http://www.ufrgs.br/agronomia/fitossan/ herbariovirtual>. Acesso em: 02 mar 2018.

EDGINGTON, L. V.; KHEW, K. L.; BARRON, G. L. Fungitoxic spectrum of benzimidazoles compounds, Phytopathology, Saint Paul, v. 61, p. 42-44, 1971.

FERREIRA, D. F. Sisvar. Versão 5.6. Lavras: UFLA/DEX, 2015. Disponível em: <http://www.dex.ufla.br/>. Acesso em: 02 de Setembro de 2017.

FRAC_FUNGICIDE RESISTANCE ACTION COMMITTEE. 2015. Disponível em: <http://www.frac.info/home. Acesso em: 10 de Jan. de 2018.

GHINI, R.; KIMATI, H. Resistência de fungos a fungicidas. 1. ed. Jaguariúna: Embrapa Meio Ambiente, 2000. $78 \mathrm{p}$

GOES, A. Queda prematura de Citrus: caracterização do agente causal, Colletotrichum gloeosporioides PENZ [Senso ARX, 1957, e controle da doença]. 1995. 16f. Tese (Doutorado em Fitopatologia) - Escola
Superior de Agricultura "Luis de Queiroz", Piracicaba, 1995.

JAYASINGHE, C. K.; FERNANDO, T. H. P. S. Growth at different temperatures and on fungicide amended media: Two characteristics to distinguish Colletotrichum species pathogenic to rubber. Mycopathologia, Den Haag, v. 143, n. 2, p. 93-95, 1998. DOI: https://dx.doi.org/10.1023/A:1006958623733

KHAN, S. H.; AKHED, J.; MAGAN, N. Control of the anthracnose pathogen of banana (Colletotrichum musae) using antioxidants alone and in combination with thiabendazole or imazalil. Plant Pathology, Honolulu, v. 50, p. 601-608, 2001.

KIMATI, H.; AMORIM, L.; REZENDE, J.A.M.; BERGAMIM FILHO, A.; CAMARGO, L.E.A. Manual de fitopatologia: doenças das plantas cultivadas. São Paulo: Ceres, 2005. 2 v. 663 p.

KOSOSKI, R. M.; FURLANETTO, C.; TOMITA, C. K.; CAFÉ FILHO, A. C. Efeito de fungicidas em Colletotrichum acutatum em morango e controle da antracnose em morangueiro. Fitopatologia Brasileira, Brasília, v. 26, n. 3, p. 662-666, 2001. DOI: http://dx.doi.org/10.1590/S0100-41582001000300016

KUPPER, K. C.; GIMENES-FERNANDES, N. Isolamento e seleção de Bacillus spp. para o controle de Colletotrichum acutatum em flores destacadas de lima ácida 'Tahiti'. Summa Phytopathologica, Botucatu, v. 28, n. 3, p. 292-295, 2002.

LAPEYRE DE BELAIRE, L. de; DUBOIS, C. Distribution of thiabendazole- resistance Colletotrichum musae isolates from Guadalupe banana plantations. Plant Disease, Saint Paul, v. 81, n. 12, p. 1378-1383, 1997.

LIYANAGE, H. D.; MCMILLAN R. T.; KISTLER H. C. Two genetically distinct populations of Colletotrichum gloeosporioides from citrus. Phytopathology, Saint Paul, v. 82 p.1371-1376, 1992.

MAHADTANAPUK, S.; YU, L. D.; CUTLER, R.; VILAITHONG, T.; ANUNTALABHOCHAI, S. Mutation of Bacillus licheniformis using low-energy ion bean bombardmet. Surface Coatings Technology, v. 201, n. 19-20, p. 8028-8033, 2007.

MEDINA, M. M.; PEREIRA, M. E. C. Pós-Colheita. In: BORGES, A. L.; SOUZA, L. S. O cultivo da bananeira. 2004. p. 209-231.

MAPA_MINISTÉRIO DA AGRICULTURA, PECUÁRIA E ABASTECIMENTO. AGROFIT. Sistema de Agrotóxicos Fitossanitários. Consulta de Praga/Doença. Brasília: Ministério da Agricultura, Pecuária e Abastecimento/Coordenação-Geral de Agrotóxicos e Afins, 2011. Disponível em: http://extranet.agricultura.gov.br/agrofit_cons/principa 1_agrofit_cons. Acesso em: 16 abr. 2018.

PARREIRA, D. F.; NEVES, W. S.; ZAMBOLIM, L. Resistência de fungos a fungicidas inibidores de quinona. Revista Tropica: Ciências Agrárias e Biológicas, Chapadinha. v. 3, n. 2, p. 24-34, 2009.

PEREIRA, A. V. S. Sensibilidade a fungicidas e adaptabilidade de Lasiodiplodia theobromae patogênico ao mamão. 2009. 57f. Dissertação (Mestrado em Fitopatologia) - Universidade Federal Rural de Pernambuco, 2009.

PUPO, M. S.; ALVES, E. S. S.; SANTOS, R. B.; VENTURA, J. A.; FERNANDES, P. M. B. Antifungal activity of monoterpenes against the plant pathogens Collethotrichum 
gloesporioides, Colletotrichum musae, Fusarium subglutinans f.sp. ananas. Applied and Environmental Microbiology submitted, 2003.

REIS, E. M.; REIS. A. C.; CARMONA, M. A. Manual de fungicidas - Guia para o Controle Químico de Doenças de plantas. 6. ed. Passo Fundo: Editora UPF, 2010. 226 p.

SILVA, M. B.; COSTA, A. S. V. da; RUFINI, J. C. M.; GALVÃO, E. R.; ZAMBOLIM, L. Tratamento térmico e prochloraz no controle da antracnose em póscolheita de frutos de banana "Prata-Anã". Summa Phytopathologica, Botucatu, v. 34, n. 4, p. 364-5, 2008. DOI: http://dx.doi.org/10.1590/S010054052008000400013

STRACIERI, J. Caracterização morfocultural e molecular de Colletotrichum spp. associados a antracnose em manga, mamão e goiaba. 2015. $109 f$. Tese (Doutorado em Produção Vegetal) - Universidade Estadual Paulista, Jaboticabal, 2015.

TAVARES, G. M. Controle químico e hidrotérmico da antracnose em frutos de mamoeiro (Carica papaya L.) na pós-colheita. 2004. 55f. Dissertação (Mestrado em Fitopatologia) - Departamento de Fitopatologia, Universidade Federal de Lavras, Lavras, 2004.
TAVARES, G. M.; SOUZA, P. E. Efeito de fungicidas no controle in vitro de Colletotrichum gloeosporioides, agente etiológico da antracnose do mamoeiro (Carica papaya L.). Ciência e Agrotecnologia, Lavras, v. 29, n. 1, p. 52-59, $2005 . \quad$ DOI: http://dx.doi.org/10.1590/S1413-70542005000100006

VALDEBENITO-SANHUEZA, R. M.; CATTANIO, M. E. Controle biológico de Penicillium expansum em pós colheita de maçãs 'Fuji'. Fitopatologia Brasileira, Jabuticabal, v.26, p.445. 2001.

VENTURA, J. A.; HINZ, R. H. Controle das doenças da bananeira. In: ZAMBOLIM, L.; VALE, F. X. R. do; MONTEIRO, A. J. A.; COSTA, H. Controle de doenças de plantas fruteiras. Viçosa: UFV, 2002. v. 1. p. 838-938.

VENTUROSO, L. R.; BACCHI, L. M. A.; GAVASSONI, W. L.; PONTIM, B. C. A.; CONUS, L. A. Influência de diferentes metodologias de esterilização sobre a atividade antifúngica de extratos aquosos de plantas medicinais. Revista Brasileira de Plantas Medicinais, v.12, n.4, p.499-505, 2010. 